\title{
Guest editorial: special issue on real time and network systems
}

\author{
Liliana Cucu-Grosjean ${ }^{1}$ - Nathan Fisher ${ }^{2}$
}

Published online: 21 May 2018

(C) Springer Science+Business Media, LLC, part of Springer Nature 2018

Recent technological trends such as the internet-of-things, autonomous vehicles, and cyber-physical systems are all built upon interconnected embedded systems. Furthermore, these innovations often require formal temporal verification to ensure the safe operation of the system. Thus, there is a compelling need for new scheduling techniques and associated analysis that can address temporal properties of these networked, realtime embedded systems. The International Conference on Real-Time Networks and Systems (RTNS) provides an appropriate venue for sharing new ideas, experiences and information among academic researchers, developers and service providers in the field of real-time systems and networks. In 2015, the 23rd edition of RTNS was held in Lille, France with a diverse program representing a selection of 31 papers (from a total of 66 submissions) describing state-of-the-art results in multiple topics on real-time and network systems: e.g., real-time system design and analysis (task and message scheduling, modelling, verification, evaluation, model-driven development, worst-case execution time estimation, distributed systems, fault tolerance, quality of service, security), infrastructure and hardware for real-time systems (wired and wireless communication networks, fieldbuses, networked control systems, control/computing co-design, sensor networks, power-aware techniques), software technologies (real-time compilers, programming languages, middleware and component-based technologies, operating systems, databases) and their applications in domains like automotive, avionics, space, railways, telecommunications, process control and multimedia.

\footnotetext{
$凶 \quad$ Nathan Fisher

fishern@wayne.edu

Liliana Cucu-Grosjean

liliana.cucu@inria.fr

1 INRIA, Paris, France

2 Wayne State University, Detroit, USA
} 
Papers recognized as outstanding papers from RTNS 2015 were invited to submit extended journal versions for this special issue of the Real-Time Systems Journal. In total, we received four submissions that are presented in this issue after rigorous peer review by experts from the areas of real-time and networked systems. This issue presents a sample of significant new results, techniques, models, and analysis for real-time and networked systems as well as industry understanding on these advances.

The first work in this special issue by Robert I. Davis, Sebastian Altmeyer, Leandro S. Indrusiak, Claire Maiza, Vincent Nélis, and Jan Reineke addresses an important and pressing challenge in embedded system design: bounding response time for multicore platforms while considering the contention due to the memory hierarchy. The authors propose a flexible framework that permits formulation of a task's response time as a function of the hardware configuration. The framework can be employed at design time to search for the best configurations to meet the system's temporal demands.

The second paper by Guillaume Phavorin, Pascal Richard, Joël Goossens, Claire Maiza, Laurent George, and Thomas Chapeaux investigates the effects of delays due to preeemptions upon hard real-time systems. Fundamentally, the authors show that optimal online algorithms do not exist in the presence of preemption delays (if future job arrivals are not known in advance). In light of this provably limitation, the authors propose optimal offline solutions and investigate the effect of preemption on (nonoptimal) online scheduling algorithms.

The third paper by Sahar Abbaspour, Florian Brandner, Amine Naji and Mathieu Jan studies the impact of cache preemption delays; more precisely, the authors propose an efficient context switching solution based on a reorganization of the stack cache. The associated analysis provides an impressive gain while saving the task context, and it opens the way for new schedulability analyses.

The final paper by Alexandre Esper, Geoffrey Nelissen, Vincent Nélis and Eduardo Tovar compares two views of the mixed-criticality concept from industry point of view and from the academic one. The paper underlines the possible means to bridge the gap between the two worlds. Both industry and academic readers have the opportunity here to get a better understanding of the respective viewpoints on mixed-criticality.

We would like to thank all the authors that submitted their work to the special issue and express appreciation to the referees for their time and effort in selecting and improving the quality of the papers. 